\title{
On the Pricing of Inflation-Indexed Caps
}

\author{
Susanne Kruse K** $^{* *}$ \\ ${ }^{*}$ Hochschule der Sparkassen-Finanzgruppe - University of Applied Sciences - Bonn, Ger- \\ many. \\ ${ }^{* *}$ Fraunhofer Institute for Industrial and Financial Mathematics, Department of Finan- \\ cial Mathematics, Kaiserslautern, Germany.
}

\begin{abstract}
We consider the problem of pricing inflation-linked caplets in a BlackScholes-type framework as well as in the presence of stochastic volatility. By using results on the pricing of forward starting options in Heston's Model on stochastic volatility, we derive closed-form solutions for inflations caps which aim to receive smile-consistent option prices. Additionally we price options on the inflation development over a longer time horizon. In this paper we develop a new and more suitable formula for pricing inflation-linked options under the assumption of stochastic volatility. The formula in the presence of stochastic volatility allows to cover the smile effects observed in our Black-Scholes type environment, in which the exposure of year-on-year inflation caps to inflation volatility changes is ignored. The chosen diffusion processes reflect the macro-economic concept of Fisher making a connection between interest rates on the market and the expected inflation rate.
\end{abstract}

Key words: Inflation-indexed options, year-on-year inflation caps, Heston model, stochastic volatility, option pricing

JEL Classification: G13

Mathematics Subject Classification (1991): 91B28, 91B70, 60G44, 60H30 


\section{Introduction}

The recent evolution on stock and bond markets has shown that neither stocks nor bonds inherit an effective protection against the loss of purchasing power. Declining stock prices and a comparatively low interest rate level were and are not able to offer an effective compensation for the existing inflation rate. One characteristic of inflation is the lasting and overall rise in prices leading to a loss in the purchasing power of the money. Inflation is usually measured by a consumer price index (CPI) which is reflecting the actual price level of a basket of typical consumer goods. In the eurozone this is a harmonized consumer price index (HCPI) determined by EUROSTAT. EUROSTAT's Monetary Union Index of Consumer Prices (MUICP) is the weighted average of the specific nationals HCPIs of the eurozone where the weights rely on the national proportion of the overwhole expenses on consumption. Inflation itself is then defined as the percentage change of the inflation index, the so-called inflation rate. No matter what causes inflation - a rise in prices in foreign countries, rising costs, increasing government debt or a growing demand on consumption goods - central banks play a major role in stabilization policy. Furthermore investors ask for a compensation to cover inflation when investing into bonds - hence there is an obvious connection between the interest rates on the market and expectations on the future inflation rate. One theory reflecting this relation dates back to Fisher (1930) who describes the relation between market interest rates and inflation by the so-called Fisher Equation

$$
r_{N}(t, T)=r_{R}(t, T)+\mathbb{E}[i(t, T)]
$$

where $r_{N}(t, T)$ is the today's cumulative nominal interest rate from $t$ up to $T$ on the market, $r_{R}(t, T)$ is the real cumulative interest rate, the gain in wealth an investor wants for an investment up to maturity $T$ excluding the loss of purchasing power since time $t$ and $\mathbb{E}[i(t, T)]$ is the expected overall inflation rate from $t$ up to time $T$. We note that in the presence of continuous annual interest rates the Fisher equation rewrites as follows

$$
e^{\left(r_{N}-r_{R}\right)(T-t)}=\mathbb{E}\left[\frac{I(T)}{I(t)}\right]
$$

where $r_{N}$ is the constant continuous nominal interest rate, $r_{R}$ the constant continuous real interest rate and $I(t)$ the inflation index at time $t$ taking into account that the relation between the inflation index $I$ and the inflation rate $i$ is given by

$$
i(t, T)=\frac{I(T)-I(t)}{I(t)} .
$$

On the background of the latest issues of inflation-linked government bonds in the eurozone* and a growing number of inflation-linked life insurance products the market for inflation-linked derivatives is expected to grow. These derivatives are designed to protect investors against the changes in purchasing power of a

\footnotetext{
* Germany has issued its first inflation-linked bond with maturity in 2016 in March 2006 and a second one with maturity 2013 in October 2007.
} 
certain country or a certain economic region such as the eurozone. In addition to inflation-linked bonds these derivatives cover up the risk of inflation - or even deflation - and can be used to hedge future cash flows against inflation risk.

Inflation-linked derivatives are usually priced using a foreign currency analogy. Nominal values can be transfered into real values in purchasing power by using the value of the quotient of the two relevant inflation indices as an exchange rate. In these frameworks the nominal interest rates refer to the "domestic currency" while the real can be concerned as the "foreign currency" or vice versa. We note that this analogy does not take into account, that today's real rates on the market differ from the actual later realized gain in purchasing power.

Hugston (1998) introduced a Heath-Jarrow Morton (HJM) framework modelling the nominal and real bond prices and achieves a closed-form solution for vanilla options on the actual inflation. Jarrow and Yildirim (2003) also used a HJM framework to derive the price of a plain vanilla call on the inflation index. Jarrow and Yildirim assumed the nominal and real rates both to follow one-factor Gaussian processes along with a lognormal CPI preserving the Fisher equation. A simple model introducing a similar setting to Jarrow and Yildirim (2003) can be found in Korn and Kruse (2004) also preserving the Fisher Equation. Alternative approaches using lognormal forward CPI have been introduced by Kazziha (1999), Belgrade et al. (2004) and Mercurio (2005). These authors assume that the forward CPIs follow driftless Brownian motions under the relevant forward measures. Mercurio and Moreni (2006) extend these lognormal forward CPI models to stochastic volatility. The macroeconomic concept of Fisher is not reflected by those lognormal forward CPI models.

Through out this paper we consider the problem of pricing vanilla options on the actual inflation rate as well as the pricing of inflation caplets. Based on the Black-Scholes type model of Korn and Kruse (2004) we derive the price of a caplet on the future inflation and extend our market model to stochastic volatility as in Heston (1993). Other well known models of stochastic volatility can be found in Hull and White (1987) and Stein and Stein (1991), a good overview is given in Fouque et al. (2000). Since we believe the Heston model to be of significant importance to practioners we chose this framework to introduce our new model for inflation. Critical discussion of Heston's model as in Quesette (2002) seem to have been overcome. Kruse and Nögel (2005) show that it can be satisfactorily fitted to market data and very well reproduces implied Black-Scholes volatilities and how one can avoid critical implementation problems. We note that a detailed description of the implementation procedure can be found in Mikhailov and Nögel (2003).

The main contributions of this paper are the following:

1. In the simple Black-Scholes framework as in Korn and Kruse (2004) we derive the price of a caplet on the inflation over a future time interval. 
2. By extending the Black-Scholes framework for the inflation index to the presence of stochastic volatility we introduce a new model for inflation preserving the Fisher equation.

3. By using Heston's work on stock options we state the price of a plain vanilla call on the actual inflation rate.

4. We derive a closed-form solution to the pricing problem of options on the future inflation rate by using the analogy to the pricing of forward starting options in the presence of stochastic volatility.

The remainder of the paper is structured as follows: We recall the model of Korn and Kruse (2004) and deduce a pricing formula for inflation caplets in their setting in Section 2. To illustrate the usefulness of introducing stochastic volatility we calibrate this model to a set of market data and show that the implied volatilities in the Black-Scholes type pricing formula are anything but constant over all strikes and maturities. Section 4 briefly recalls the Heston model and some results on the pricing of forward starting options in this model. Additionally we introduce to our inflation model stochastic volatility as in Heston (1993) to derive in Section 5 closed-form solutions for both types of inflation options under the assumption of stochastic volatility. Since inflation caps and floors are European type options one can easily deduce the corresponding floor prices in both models.

\section{Pricing inflation options in a Black-Scholes- type framework}

In this section we consider the simple model of Korn and Kruse (2004) where the inflation index $I(t)$ follows a geometric Brownian motion

$$
d I(t)=\left(r_{N}-r_{R}\right) I(t) d t+\sigma_{I} I(t) d W_{I}(t)
$$

and $(\Omega, \mathcal{F}, Q)$ is a probability space with the filtration $\left(\mathcal{F}_{t}\right)_{t>0}$ of market information. It is quite obvious that this model preserves the Fisher equation:

$$
\mathbb{E}_{Q}\left[\frac{I(T)}{I(t)} \mid \mathcal{F}_{t}\right]=e^{\left(r_{N}-r_{R}\right)(T-t)}
$$

In their paper Korn and Kruse (2004) derived a closed-form solution for a call on the future inflation index:

Lemma 2.1. Under the assumption that the inflation index follows the dynamics given in (4) the price of a plain vanilla call option on the inflation index itself with strike price $K$, maturity $T$ and with payoff

$$
C(T, I(T))=(I(T)-K)^{+}
$$

at maturity, is at time $t=0$ given by 


$$
C(0, I(0))=I(0) e^{-r_{R} T} N(d)-K e^{-r_{N} T} N\left(d-\sigma_{I} \sqrt{T}\right)
$$

with

$$
d=\frac{\ln \left(\frac{I(0)}{K}\right)+\left(r_{N}-r_{R}+\frac{1}{2} \sigma_{I}^{2}\right) T}{\sigma_{I} \sqrt{T}} .
$$

From this result we can directly deduce the price of an option on the inflation rate starting at some time before or at the valuation date of the very same option:

Corollary 2.2. Assuming that the dynamics of the inflation index is given by (4), the price of a cap on the overall inflation rate over a fixed time horizon from time $t_{0}$ up to the maturity of the cap $T$ with $t_{0} \leq 0<T$ as in

$$
C_{I D}(T ; I(T))=\left(\frac{I(T)-I\left(t_{0}\right)}{I\left(t_{0}\right)}-k\right)^{+}
$$

is at time $t=0$ given by

$$
C_{I D}(0, I(0))=\frac{I(0)}{I\left(t_{0}\right)} e^{-r_{R} T} N(d)-(1+k) e^{-r_{N} T} N\left(d-\sigma_{I} \sqrt{T}\right)
$$

with

$$
d=\frac{\ln \left(\frac{I(0)}{I\left(t_{0}\right)(1+k)}\right)+\left(r_{N}-r_{R}+\frac{1}{2} \sigma_{I}^{2}\right) T}{\sigma_{I} \sqrt{T}} .
$$

Proof: The result is quite obvious since the inflation index $I\left(t_{0}\right)$ is a known constant at time $t=0$ and setting the strike equal to $K=I\left(t_{0}\right) \cdot(1+k)$ in Equation (5) leads to the above.

Analogously to the derivation of the price of a forward starting option in the Black-Scholes framework for stock options as in Rubinstein(1991) we achieve a closed-form formula for the price of a caplet on the inflation rate over a future time interval $\left[T_{i-1}, T_{i}\right]$ :

Proposition 2.3. Assuming that the dynamics of the inflation index is given by (4), the price of a caplet on the inflation rate $i\left(T_{i-1}, T_{i}\right)$ over a future time interval from time $T_{i-1}$ up to the maturity of the caplet at time $T_{i}$ with $0<T_{i-1}<T_{i}$ and payoff at time $T_{i}$

$$
C_{F I R}\left(T_{i} ; i\left(T_{i-1} ; T_{i}\right)\right)=\left(i\left(T_{i-1}, T_{i}\right)-k\right)^{+}=\left(\frac{I\left(T_{i}\right)-I\left(T_{i-1}\right)}{I\left(T_{i-1}\right)}-k\right)^{+}
$$


is at time $t=0$ given by

$$
\begin{aligned}
C_{F I R}(0, I(0))= & e^{-r_{R}\left(T_{i}-T_{i-1}\right)} e^{-r_{N} T_{i-1}} N(d) \\
& -(1+k) e^{-r_{N} T_{i}} N\left(d-\sigma_{I} \sqrt{T_{i}-T_{i-1}}\right)
\end{aligned}
$$

with

$$
d=\frac{-\ln (1+k)+\left(r_{N}-r_{R}+\frac{1}{2} \sigma_{I}^{2}\right) \cdot\left(T_{i}-T_{i-1}\right)}{\sigma_{I} \sqrt{T_{i}-T_{i-1}}}
$$

Proof: General arbitrage theory yields that today's option price is given by

$$
C_{F I R}(0, I(0))=\mathbb{E}_{Q}\left[\frac{B(0)\left(\frac{I\left(T_{i}\right)}{I\left(T_{i-1}\right)}-K\right)^{+}}{B\left(T_{i}\right)} \mid \mathcal{F}_{0}\right]
$$

with $K=1+k, Q$ the equivalent martingale measure and numeraire $B(t)=$ $e^{r_{N} t}$ for $t \geq 0$. As the underlying inflation index process is adapted to the filtration of market information and the numeraire is deterministic we can rewrite the option price as

$$
\mathbb{E}_{Q}\left[\frac{B(0)}{B\left(T_{i-1}\right)} \cdot \mathbb{E}_{Q}\left[\frac{B\left(T_{i-1}\right)\left(\frac{I\left(T_{i}\right)}{I\left(T_{i-1}\right)}-K\right)^{+} \mid}{B\left(T_{i}\right)} \mid \mathcal{F}_{T_{i-1}}\right] \mid \mathcal{F}_{0}\right]
$$

Since the inflation index $I\left(T_{i-1}\right)$ is a constant at time $T_{i-1}$ we can use Lemma 2.1 to derive that the inner expectation - the option price in $T_{i-1}$ - is equal to

$$
\begin{aligned}
& \mathbb{E}_{Q}\left[\frac{B\left(T_{i-1}\right)\left(\frac{I\left(T_{i}\right)}{I\left(T_{i-1}\right)}-K\right)^{+} \mid}{B\left(T_{i}\right)} \mid \mathcal{F}_{T_{i-1}}\right] \\
& =\frac{1}{I\left(T_{i-1}\right)} C\left(T_{i-1}, I\left(T_{i-1}\right)\right) \\
& =e^{-r_{R}\left(T_{i}-T_{i-1}\right)} N\left(d\left(T_{i-1}\right)\right)-K \cdot e^{-r_{N}\left(T_{i}-T_{i-1}\right)} N\left(d\left(T_{i-1}\right)-\sigma_{I} \sqrt{T_{i}-T_{i-1}}\right)
\end{aligned}
$$

with

$$
d\left(T_{i-1}\right)=\frac{\ln \left(\frac{1}{K}\right)+\left(r_{N}-r_{R}+\frac{1}{2} \sigma_{I}^{2}\right)\left(T_{i}-T_{i-1}\right)}{\sigma_{I} \sqrt{T_{i}-T_{i-1}}} .
$$

We directly get that the option price at time $t=0$ is given by

$$
\begin{aligned}
C_{F I R}(0, I(0))= & \mathbb{E}_{Q}\left[\frac{B(0)}{B\left(T_{i-1}\right)} \cdot \frac{1}{I\left(T_{i-1}\right)} C\left(T_{i-1}, I\left(T_{i-1}\right)\right) \mid \mathcal{F}_{0}\right] \\
= & e^{-r_{R}\left(T_{i}-T_{i-1}\right)} \cdot e^{-r_{N} T_{i-1}} N\left(d\left(T_{i-1}\right)\right) \\
& -K \cdot e^{-r_{N} T_{i}} N\left(d\left(T_{i-1}\right)-\sigma_{I} \sqrt{T_{i}-T_{i-1}}\right)
\end{aligned}
$$


taking into account that $d\left(T_{i-1}\right)$ is deterministic and independent of $I\left(T_{i-1}\right)$.

We note that this model is quite simple and easy to use for practioners but inherits the same assumptions as the Black-Scholes model for stock options - such as the assumption of a constant volatility over time and all strikes - that have proved to be unrealistic. Intuitively the price of an option on the inflation rate over a future time interval is depending on today's market position on the future behaviour of inflation. This is reflected by the implied forward volatilities in this Black-Scholes-type framework. In Section 3 we extract forward volatilites from a variety of different cap prices and thereby show that inflation volatility is anything than constant over all maturities and strikes.

\section{Calibration of the Black-Scholes Type Model to Market Data}

In this section we give an application of our Black-Scholes type model to market data and show that forward implied volatilities in our Black-Scholes-type model are anything but constant over all maturities and strikes. The model was calibrated to inflation-indexed cap prices for different strikes and maturities as of November 3,2004 , in the USD market as shown in Table 1 (see also Mercurio and Moreni $(2006))$.

Table 1: Cap prices (in bps) of November 3, 2004, USD market

\begin{tabular}{lllllll}
\hline$T_{i} /$ Strike & $1 \%$ & $1,50 \%$ & $2 \%$ & $2,50 \%$ & $3 \%$ & $3,50 \%$ \\
\hline 1 & 178,10 & 134,40 & 95,10 & 62,40 & 37,80 & 21,20 \\
2 & 360,40 & 277,40 & 202,90 & 140,30 & 92,00 & 58,00 \\
3 & 539,90 & 419,90 & 312,10 & 221,10 & 150,20 & 99,30 \\
4 & 714,50 & 558,90 & 418,90 & 300,40 & 207,40 & 140,00 \\
5 & 886,40 & 696,10 & 524,30 & 378,30 & 263,10 & 179,00 \\
6 & 1041,70 & 819,50 & 619,00 & 448,30 & 313,40 & 214,60 \\
7 & 1196,80 & 944,70 & 717,10 & 523,00 & 368,80 & 255,10 \\
8 & 1338,80 & 1059,50 & 807,50 & 592,30 & 420,90 & 293,80 \\
9 & 1477,40 & 1172,60 & 897,60 & 662,40 & 474,60 & 334,60 \\
10 & 1610,50 & 1281,90 & 985,40 & 731,60 & 528,30 & 376,00 \\
\hline
\end{tabular}

The real discount factors needed for both models can be directly extracted from the given rates of zero coupon inflation swaps and from the nominal discount factors as in Table 2 and where we can defined the nominal discount factors $D_{r_{N}}\left(0, T_{i}\right):=e^{-r_{N} T_{i}}$, and the real discount factors $D_{r_{R}}\left(0, T_{i}\right):=e^{-r_{R} T_{i}}$, as well 
Table 2: USD nominal and real discount factors, ZC swap rates, on November 3, 2004

\begin{tabular}{llll}
\hline$T_{i}$ & $D_{r_{N}}\left(0, T_{i}\right)$ & ZC Rates & $D_{r_{R}}\left(0, T_{i}\right)$ \\
\hline 1 & 0,97701 & $2,111 \%$ & 0,99763 \\
2 & 0,94982 & $2,188 \%$ & 0,99184 \\
3 & 0,91835 & $2,240 \%$ & 0,98146 \\
4 & 0,88433 & $2,278 \%$ & 0,96771 \\
5 & 0,84862 & $2,293 \%$ & 0,95048 \\
6 & 0,81179 & $2,300 \%$ & 0,93046 \\
7 & 0,77460 & $2,310 \%$ & 0,90887 \\
8 & 0,73785 & $2,320 \%$ & 0,88645 \\
9 & 0,70218 & $2,325 \%$ & 0,86354 \\
10 & 0,66773 & $2,335 \%$ & 0,84109 \\
\hline
\end{tabular}

as the implied nominal forward $F_{r_{N}}\left(T_{i-1}, T_{i}\right):=e^{-r_{N}\left(T_{i}-T_{i-1}\right)}$ and the implied real forward discount factors $F_{r_{R}}\left(T_{i-1}, T_{i}\right):=e^{-r_{R}\left(T_{i}-T_{i-1}\right)}$.

With the pricing formulae (7) at hand, we are able to derive the implied volatility surface as in Figure 1. At first glance one can see, that the implied volatilities are far from being constant. The special shape, i.e. the skewness of the surface gave us the confidence to continue with the derivation of an inflation caplet price in a stochastic volatility model of inflation.

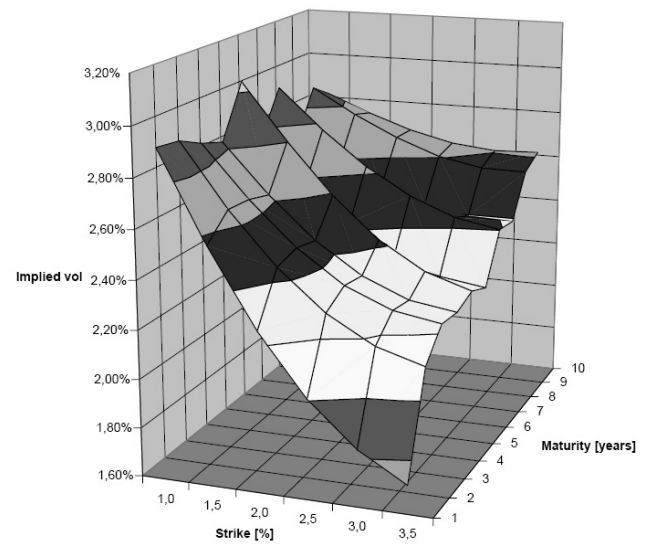

Figure 1: Implied volatility surface for inflation caplets in our Black-Scholes type model 


\section{The Heston Model}

In this section we consider a Heston framework on stochastic volatility as first introduced in Heston (1993). Let $(\Omega, \mathcal{F}, Q)$ be a probability space with filtration $\left(\mathcal{F}_{t}\right)_{t \geq 0}$ of market information with the risk neutral equivalent martingale measure $Q$ derived by calibrating to a set of given market data. We assume that the asset price process $A(t)$ follows the diffusion

$$
d A(t)=r A(t) d t+\sqrt{\nu(t)} A(t) d W_{A}(t)
$$

with constant risk free nominal interest rate $r$ while the variance $\nu(t)$ satisfies the stochastic differential equation

$$
d \nu(t)=\kappa(\theta-\nu(t)) d t+\sigma \sqrt{\nu(t)} d\left(\rho W_{A}(t)+\sqrt{1-\rho^{2}} W_{\nu}(t)\right)
$$

with risk neutral long term mean $\theta$, risk neutral mean reverting speed $\kappa$ with $\kappa \geq 0$ and volatility $\sigma$ of the variance process itself. Furthermore we suppose that $W_{A}$ and $W_{\nu}$ are independent Brownian motions with respect to the filtration $\left(\mathcal{F}_{t}\right)_{t \geq 0}$ and that the above parameters are such that the stability condition

$$
\frac{2 \kappa \theta}{\sigma^{2}}>1
$$

holds. This condition ensures that the variance process $\nu(t)$ remains positive starting from a positive variance $\nu(0)$ (for further discussion see Brigo and Mercurio (2006), pp. 56f).

Heston (1993) shows that for a plain vanilla call its option price at some time $t$ prior to maturity $T$ can be calculated as in the following lemma. We note that we work under the risk neutral measure and thereby neglect the market price of risk. Due to the partial differential equation approach to option pricing, one derives probabilities $P_{1}$ and $P_{2}$ which can be calculated by applying Fourier transformation, in other words by finding solutions to the corresponding partial differential equations for their characteristic functions. A detailed derivation of the Heston formula can for example be found in Primm (2007). We note that Heston's result can be extended to the presence of a constant dividend yield of the asset $A$ such that (8) rewrites

$$
d A(t)=(r-d) A(t) d t+\sqrt{\nu(t)} A(t) d W_{A}(t) .
$$

Lemma 3.1. Suppose that the asset price process A(t) satisfies Equation (11) and (9) such that condition (10) holds. Note that $i=\sqrt{-1}$. The price of an option with payoff

$$
C(T, A(T))=(A(T)-K)^{+}
$$

at time $t=0$ is given by

$$
\begin{aligned}
& C(0, A(0), \nu(0)) \\
& =A(0) e^{-d T} P_{1}\left(0, e^{-d T} A(0), \nu(0), K\right)-K e^{-r T} P_{2}\left(0, e^{-d T} A(0), \nu(0), K\right)
\end{aligned}
$$


where $P_{1}$ and $P_{2}$ are probabilities given by

$$
P_{j}\left(0, e^{-d T} A(0), \nu(0), K\right)=\frac{1}{2}+\frac{1}{\pi} \int_{0}^{\infty} \operatorname{Re}\left(\frac{e^{-i \phi \ln (K)} f_{j}(d, A(0), \nu(0), T, \phi)}{i \phi}\right) d \phi
$$

with $f_{j}$ being the corresponding characteristic functions defined by

$$
f_{j}(d, A(0), \nu(0), T, \phi)=e^{-d i \phi T+i \phi l n(A(0))+C_{j}(\phi, T)+D_{j}(\phi, T) \nu(0)}
$$

for

$$
\begin{gathered}
C_{j}(\phi, T)=\operatorname{ri\phi } T+\frac{\kappa \theta}{\sigma^{2}}\left[\left(b_{j}-\rho \sigma i \phi+d_{j}\right) T-2 \ln \left(\frac{1-q_{j} e^{d_{j} T}}{1-q_{j}}\right)\right] \\
D_{j}(\phi, T)=\frac{b_{j}-\rho \sigma i \phi+d_{j}}{\sigma^{2}}\left(\frac{1-e^{d_{j} T}}{1-q_{j} e^{d_{j} T}}\right)
\end{gathered}
$$

where

$$
\begin{gathered}
u_{1}=-u_{2}=\frac{1}{2}, \\
b_{1}=\kappa-\sigma \rho, \quad b_{2}=\kappa, \\
d_{j}=\sqrt{\left(\rho \sigma i \phi-b_{j}\right)^{2}-\sigma^{2}\left(2 u_{j} i \phi-\phi^{2}\right)},
\end{gathered}
$$

and

$$
q_{j}=\frac{b_{j}-\rho \sigma i \phi+d_{j}}{b_{j}-\rho \sigma i \phi-d_{j}}
$$

for $j=1,2$.

We now recall a useful result on the pricing of forward starting options in the Heston model. Forward starting options are exotic options whose strike prices are not fully determined until an intermediate date $t^{*}$ before maturity $T$, called the determination time of the strike or starting date of the option. Kruse and Nögel (2005) derive a closed-form solution to the pricing of forward starting options on the return of an asset $A$ with payoff

$$
C(A(T))=\left(\frac{A(T)}{A\left(t^{*}\right)}-k\right)^{+}
$$

in the Heston model:

Lemma 3.2. Let $0<t^{*}<T$ and suppose $\kappa \geq \rho \sigma$. Assuming (11), (9), and (10) the price of an option with payoff (13) can be calculated by

$$
\begin{aligned}
C(0, \nu(0), A(0))= & e^{-d\left(T-t^{*}\right)} e^{-r t^{*}} \hat{P}_{1}\left(e^{-d\left(T-t^{*}\right)}, 0, \nu(0)\right) \\
& \left.-k e^{-r T} \hat{P}_{2}\left(e^{-d\left(T-t^{*}\right)}, 0, \nu(0)\right)\right) .
\end{aligned}
$$


where $P_{1}$ and $P_{2}$ are probabilities defined by

$$
\hat{P}_{j}(x, 0, \nu(0)):=\int_{0}^{\infty} P_{j}\left(t^{*}, x, v, k\right) f(v \mid \nu(0)) d v
$$

where the $P_{j}, j=1,2$, equal the Heston probabilities given in (12) and

$$
f(v \mid \nu(0))=\frac{B}{2} \cdot e^{-(B v+\Lambda) / 2}\left(\frac{B v}{\Lambda}\right)^{(R / 2-1) / 2} I_{R / 2-1}(\sqrt{\Lambda B v})
$$

for

$$
\Lambda=B e^{-(\kappa-\rho \sigma) t^{*}} \nu(0),
$$

with

$$
B=\frac{4(\kappa-\rho \sigma)}{\sigma^{2}}\left(1-e^{-(\kappa-\rho \sigma) t^{*}}\right)^{-1}
$$

and

$$
R=\frac{4 \kappa \theta}{\sigma^{2}}
$$

while $I_{R / 2-1}(\cdot)$ is a modified Bessel function of the first kind.

\section{Pricing inflation-linked caps in Heston's model on stochastic volatility}

We now consider a Heston-type framework to model the inflation index $I(t)$. Let $(\Omega, \mathcal{F}, Q)$ be a probability space with filtration $\left(\mathcal{F}_{t}\right)_{t \geq 0}$ of market information with the risk neutral equivalent martingale measure $Q$ derived by calibrating to a set of given market data. We assume that the inflation index $I(t)$ follows the diffusion

$$
d I(t)=\left(r_{N}-r_{R}\right) I(t) d t+\sqrt{\nu(t)} I(t) d W_{I}(t)
$$

with constant risk free nominal interest rate $r_{N}$ and real interest rate $r_{R}$, while the variance $\nu(t)$ satisfies the stochastic differential equation

$$
d \nu(t)=\kappa_{I}\left(\theta_{I}-\nu(t)\right) d t+\sigma_{I} \sqrt{\nu(t)} d\left(\rho W_{I}(t)+\sqrt{1-\rho^{2}} W_{\nu}(t)\right)
$$

with risk neutral long term mean $\theta_{I}$, risk neutral mean reverting speed $\kappa_{I}$ with $\kappa_{I} \geq 0$ and volatility $\sigma_{I}$ of the variance process itself. Furthermore we suppose that $W_{I}$ and $W_{\nu}$ are independent Brownian motions with respect to the filtration $\left(\mathcal{F}_{t}\right)_{t \geq 0}$ and that the above parameters are such that the stability condition

$$
\frac{2 \kappa_{I} \theta_{I}}{\sigma_{I}^{2}}>1
$$

holds. As before this condition ensures that the variance process $\nu(t)$ remains positive starting from a positive variance $\nu(0)$. 
Lemma 4.1. Suppose that the inflation index $I(t)$ satisfies Equation (14) and (15) such that condition (16) holds. Again holds $i=\sqrt{-1}$. The price of an option with payoff

$$
C(T, I(T))=(I(T)-K)^{+}
$$

at time $t=0$ is given by

$$
\begin{aligned}
& C(0, I(0), \nu(0)) \\
& =I(0) e^{-r_{R} T} P_{1}\left(0, e^{-r_{R} T} I(0), \nu(0), K\right)-K e^{-r_{N} T} P_{2}\left(0, e^{-r_{R} T} I(0), \nu(0), K\right)
\end{aligned}
$$

where $P_{1}$ and $P_{2}$ are probabilities given by

$$
P_{j}\left(0, e^{-r_{R} T} I(0), \nu(0), K\right)=\frac{1}{2}+\frac{1}{\pi} \int_{0}^{\infty} \operatorname{Re}\left(\frac{e^{-i \phi \ln (K)} f_{j}\left(r_{R}, I(0), \nu(0), T, \phi\right)}{i \phi}\right) d \phi
$$

with $f_{j}$ being the corresponding characteristic functions defined by

$$
f_{j}\left(r_{R}, I(0), \nu(0), T, \phi\right)=e^{-r_{R} i \phi T+i \phi l n(I(0))+C_{j}(\phi, T)+D_{j}(\phi, T) \nu(0)}
$$

for

$$
\begin{gathered}
C_{j}(\phi, T)=r_{N} i \phi T+\frac{\kappa_{I} \theta_{I}}{\sigma_{I}^{2}}\left[\left(b_{j}-\rho \sigma_{I} i \phi+d_{j}\right) T-2 \ln \left(\frac{1-q_{j} e^{d_{j} T}}{1-q_{j}}\right)\right] \\
D_{j}(\phi, T)=\frac{b_{j}-\rho \sigma_{I} i \phi+d_{j}}{\sigma_{I}^{2}}\left(\frac{1-e^{d_{j} T}}{1-q_{j} e^{d_{j} T}}\right)
\end{gathered}
$$

where

$$
\begin{gathered}
u_{1}=-u_{2}=\frac{1}{2}, \\
b_{1}=\kappa_{I}-\sigma_{I} \rho, \quad b_{2}=\kappa_{I}, \\
d_{j}=\sqrt{\left(\rho \sigma_{I} i \phi-b_{j}\right)^{2}-\sigma_{I}^{2}\left(2 u_{j} i \phi-\phi^{2}\right)},
\end{gathered}
$$

and

$$
q_{j}=\frac{b_{j}-\rho \sigma_{I} i \phi+d_{j}}{b_{j}-\rho \sigma_{I} i \phi-d_{j}}
$$

for $j=1,2$.

Proof: This results directly from Lemma 3.1 if one regards the real interest rate $r_{R}$ as a form of dividend yield on the inflation index.

From the former result we directly achieve the price of an option on the actual inflation rate:

Corollary 4.2. Assuming that the dynamics of the inflation index is given by (14) and (15) such that condition (16) holds, the price of a cap on the overall inflation 
rate over a fixed time horizon from time $t_{0}$ up to the maturity of the cap $T$ with $t_{0} \leq 0<T$ as in

$$
C_{I D}(T ; I(T))=\left(\frac{I(T)-I\left(t_{0}\right)}{I\left(t_{0}\right)}-k\right)^{+}
$$

is at time $t=0$ given by

$$
\begin{aligned}
C_{I D}(0, I(0), \nu(0)) & =\frac{I(0)}{I\left(t_{0}\right)} e^{-r_{R} T} P_{1}\left(0, e^{-r_{R} T} I(0), \nu(0), I\left(t_{0}\right)(1+k)\right) \\
& -(1+k) e^{-r_{N} T} P_{2}\left(0, e^{-r_{R} T} I(0), \nu(0), I\left(t_{0}\right)(1+k)\right)
\end{aligned}
$$

where $P_{j}, j=1,2$ equal the Heston probabilities as in (17).

Proof: This follows directly from the above by rewriting the payoff as

$$
C_{I D}(T ; I(T))=\frac{1}{I\left(t_{0}\right)}\left(I(T)-I\left(t_{0}\right) \cdot(1+k)\right)^{+}=\frac{1}{I\left(t_{0}\right)}(I(T)-K)^{+}
$$

with $K=I\left(t_{0}\right) \cdot(1+k)$.

We can now apply Lemma 3.2 to achieve the price of a caplet on the future inflation over a time interval $\left[T_{i-1} ; T_{i}\right]$ :

Propostion 4.3. Under the same assumptions as above the price of a caplet on the inflation rate $i\left(T_{i-1}, T_{i}\right)$ over a future time interval from time $T_{i-1}$ up to the maturity of the caplet at time $T_{i}$ with $0<T_{i-1}<T_{i}$ as in

$$
C_{F I R}(T ; I(T))=\left(i\left(T_{i-1}, T_{i}\right)-k\right)^{+}=\left(\frac{I\left(T_{i}\right)-I\left(T_{i-1}\right)}{I\left(T_{i-1}\right)}-k\right)^{+}
$$

is at time $t=0$ given by

$$
\begin{aligned}
C_{F I R}(0, I(0), \nu(0)) & =e^{-r_{R}\left(T_{i}-T_{i-1}\right)} e^{-r_{N} T_{i-1}} \hat{P}_{1}\left(0, e^{-r_{R}\left(T_{i}-T_{i-1}\right)}, \nu(0), 1+k\right) \\
& -(1+k) e^{-r_{N} T_{i}} \hat{P}_{2}\left(0, e^{-r_{R}\left(T_{i}-T_{i-1}\right)}, \nu(0), 1+k\right)
\end{aligned}
$$

where the $\hat{P}_{j}, j=1,2$ are given by

$$
\begin{aligned}
\hat{P}_{j}\left(0, e^{-r_{R}\left(T_{i}-T_{i-1}\right)}, \nu(0), 1+k\right) \\
\quad:=\int_{0}^{\infty} P_{j}\left(T_{i-1}, e^{-r_{R}\left(T_{i}-T_{i-1}\right)}, v, 1+k\right) f(v \mid \nu(0)) d v
\end{aligned}
$$

where the $P_{j}, j=1,2$, equal the Heston probabilities given in (17) and

$$
f(v \mid \nu(0))=\frac{B}{2} \cdot e^{-(B v+\Lambda) / 2}\left(\frac{B v}{\Lambda}\right)^{(R / 2-1) / 2} I_{R / 2-1}(\sqrt{\Lambda B v})
$$

for

$$
\Lambda=B e^{-\left(\kappa_{I}-\rho \sigma_{I}\right) T_{i-1}} \nu(0)
$$


with

$$
B=\frac{4\left(\kappa_{I}-\rho \sigma_{I}\right)}{\sigma_{I}^{2}}\left(1-e^{-\left(\kappa_{I}-\rho \sigma_{I}\right) T_{i-1}}\right)^{-1}
$$

and

$$
R=\frac{4 \kappa_{I} \theta_{I}}{\sigma_{I}^{2}}
$$

while $I_{R / 2-1}(\cdot)$ is a modified Bessel function of the first kind.

Proof: This follows by applying the results of Kruse and Nögel (2005) as in Lemma 3.2 on forward starting options on the return of an underlying as in

$$
C_{F I R}\left(T_{i} ; I\left(T_{i}\right)\right)=\left(\frac{I\left(T_{i}\right)-I\left(T_{i-1}\right)}{I\left(T_{i-1}\right)}-k\right)^{+}=\left(\frac{I\left(T_{i}\right)}{I\left(T_{i-1}\right)}-(k+1)\right)^{+}
$$

with determination time $T_{i-1}$ and maturity $T_{i}$.

\section{Conclusions}

In this paper we constructed closed-form solutions to the pricing problem of inflation options in both a Black-Scholes-type framework as well as in a stochastic volatility model analogously to Heston (1993). Besides the pricing of options on the inflation development since today we give a closed-form solution for caplets on the future inflation rate in both models. The models introduced are reflecting the macroeconmic concept of the Fisher equation giving functional connection between the nominal and the real interest rate. The derivation of our pricing formulae hinges on the similarity between options on the future inflation rate and forward starting options on the return of an underlying. We note that our solutions in the stochastic volatility model do not depend on any assumption on the correlation between the inflation index and its variance as for instance assumed in Mercurio and Moreni (2006). Therefore it is less cumbersome to calibrate our stochastic volatility model to market prices. The main advantages of both models once implemented are the speed of evaluation and their simplicity. By extracting the implied volatilities from our Black-Scholes-type prices one can see that the implied volatilities are anything but constant over all maturities and strikes which gives us the justification for our stochastic volatility model of inflation.

Hence it is useful to introduce a stochastic variance process in order to capture the changes in volatility and its influence on option prices on the future inflation. The inflation market matures and becomes more liquid, inflation products are getting more complex and there will be more exotic inflation options coming up. A natural extension of our stochastic volatility model for inflation caps and floors is to add dynamics for the nominal as well as the real interest rate structure. 
Additionally one could also consider a jump diffusion process for the inflation index.

\section{Acknowledgement}

Furthermore, we would like to thank Fabio Mercurio, Banca IMI Milano, for offering to use the market data as in Mercurio and Moreni (2006).

\section{References}

[1] Belgrade, N., Benhamou, E. and Köhler, E. (2004): A Market Model for Inflation, SSRN Working Paper.

[2] Black, F. and Scholes, M. (1973): The pricing of options and corporate liabilities, Journal of Political Economy, 81, 637-659.

[3] Brigo, D. and Mercurio, F. (2006): Interest rate models - theory and practice, Berlin, Heidelberg, New York: Springer.

[4] Fisher, I. (1930): The Theory of Interest, London, Basingstoke: MacMillan Press Ltd.

[5] Fouque, J.P., Papanicolaou, G. and Sircar, K.R. (2000): Derivatives in Financial Markets with Stochastic Volatility, Cambridge, Madrid, Melbourne, New York: Cambridge University Press.

[6] Heston, S. (1993): A closed-form solution for options with stochastic volatility and applications to bond and currency options, Review of Financial Studies, 6 (2), 327-343.

[7] Hughston, L. (1998): Inflation Derivatives, working paper.

[8] Hull, J. and White, A. (1987): The pricing of options on assets with stochastic volatility, Journal of Finance, 42, 281-300.

[9] Jarrow, R. and Yildirim, Y. (2003): Pricing Treasury Inflation Protected Securities and Related Derivatives using an HJM model, Journal of Financial and Quantitative Analysis, 38, 409-430.

[10] Kazziha, S. (1999): Interest Rate Models, Inflation-based Derivatives, Trigger Notes and Cross-Currency Swaptions. PhD Thesis, Imperical College of Science, Technology and Medicine, London.

[11] Korn, R. and Kruse, S. (2004): A simple model to value inflation-linked financial products, (in German), Blätter der DGVFM, XXVI (3), 351-367.

[12] Kruse, S. and Nögel, U. (2005): On the pricing of forward starting options in Heston's model on stochastic volatility, Finance and Stochastics, 9, 233-250. 
[13] Mikhailov, S. and Nögel, U. (2003): Heston's Stochastic Volatility Model Implementation, Calibration and Some Extensions, WILMOTT magazine, July 2003.

[14] Mercurio, F. (2005): Pricing Inflation-Indexed Derivatives, Quantitative Finance, 5 (3), 289-302.

[15] Mercurio, F. and Moreni, N. (2006): Inflation-Indexed Securities - Inflation with a Smile, Risk, 19 (3), 70-75 (2006).

[16] Rubinstein, M. (1991): Pay Now, Choose Later. Risk, 4 (2), 13.

[17] Stein, E. and Stein, J. (1991): Stock price distributions with stochastic volatility: An analytic approach, Review of Financial Studies, 4 (4), 727-752. 